\title{
An Improvement of a Known Unique Common Fixed Point Result for Four Mappings on 2-Metric Spaces*
}

\author{
Ailian Jin, Yongjie Piao \\ Department of mathematics, College of Science, Yanbian University, Yanji, China \\ Email: "\#yj6216@hotmail.com
}

Received February 28, 2013; revised March 28, 2013; accepted April 5, 2013

Copyright (C) 2013 Ailian Jin, Yongjie Piao. This is an open access article distributed under the Creative Commons Attribution License, which permits unrestricted use, distribution, and reproduction in any medium, provided the original work is properly cited.

\begin{abstract}
In this paper, we introduce a new class $\Gamma$, which is weaker than a known class $\Psi$, of real continuous functions defined on $[0,+\infty)$, and use another method to prove the known unique common fixed point theorem for four mappings with $\gamma$-contractive condition instead of $\psi$-contractive condition on 2-metric spaces.
\end{abstract}

Keywords: 2-Metric Space; Class $\Gamma$; Class $\Psi$; Common Fixed Point

\section{Introduction}

The second author has obtained an unique common fixed point theorem for four mappings with $\psi$-contrac- tive condition [1,2] on 2-metric spaces in [1], where $\psi$ is a continuous and non-decreasing real function on $[0,+\infty)$ satisfying that $\psi(t)<t$ for all $t>0$. The re- sult generalizes and improves many corresponding results.

Here, we introduce a new class $\Gamma$ of real functions defined on $[0,+\infty)$, and reprove the well known unique common fixed point theorem for four mappings with $\psi$-contractive condition replaced by $\gamma$-contractive condition on 2-metric spaces. The method used in this paper is very different from that in [1].

At first, we give well known definitions and results.

Definition 1.1. ([3,4]) A 2-metric space $(X, d)$ consists of a nonempty set $X$ and a function

$$
d: X \times X \times X \rightarrow[0,+\infty)
$$

such that

1) for distant elements $x, y \in X$, there exists an $u \in X \quad$ such that $d(x, y, u) \neq 0$;

2) $d(x, y, z)=0$ if and only if at least two elements in $\{x, y, z\}$ are equal;

3) $d(x, y, z)=d(u, v, w)$, where $\{u, v, w\}$ is any permutation of $\{x, y, z\}$;

4) $d(x, y, z) \leq d(x, y, u)+d(x, u, z)+d(u, y, z)$ for all $x, y, z, u \in X$.

"This paper is supported by the NNSF of China (No. 11261062) and (N0. 11361064)

${ }^{\#}$ Corresponding author.
Definition 1.2. ([3,4]) A sequence $\left\{x_{n}\right\}_{n \in \mathbb{N}}$ in 2-metric space $(X, d)$ is said to be cauchy sequence, if for each $\varepsilon>0$ there exists a positive integer $N \in \mathbb{N}$ such that $d\left(x_{n}, x_{m}, a\right)<\varepsilon$ for all $a \in X$ and $n, m>N$.

Definition 1.3. ([5,6]) A sequence $\left\{x_{n}\right\}_{n \in \mathbb{N}}$ is said to be convergent to $x \in X$, if for each $a \in X$,

$$
\lim _{n \rightarrow+\infty} d\left(x_{n}, x, a\right)=0 \text {. }
$$

And write $x_{n} \rightarrow x$ and call $x$ the limit of $\left\{x_{n}\right\}_{n \in \mathbb{N}}$.

Definition 1.4. ([5,6]) A 2-metric space $(X, d)$ is said to be complete, if every cauchy sequence in $X$ is convergent.

Definition 1.5. ([7,8]) Let $f$ and $g$ be two selfmappings on a set $X$. If $w=f x=g x$ for some $x \in X$, then $x$ is called a coincidence point of $f$ and $g$, and $w$ is called a point of coincidence of $f$ and $g$.

Definition 1.6. ([9]) Two mappings $f, g: X \rightarrow X$ are said to be weakly compatible if, for every $x \in X$, holds $f g x=g f x$ whenever $f x=g x$.

The following three lemmas are known results.

Lemma 1.7. ([3-6]) Let $(X, d)$ be a 2-metric space and $\left\{x_{n}\right\}_{n \in \mathbb{N}}$ a sequence. If there exists $h \in[0,1)$ such that

$$
d\left(x_{n+2}, x_{n+1}, a\right) \leq h d\left(x_{n+1}, x_{n}, a\right)
$$

for all $a \in X$ and $n \in \mathbb{N}$, then $d\left(x_{n}, x_{m}, x_{l}\right)=0$ for all $n, m, l \in \mathbb{N}$, and $\left\{x_{n}\right\}_{n \in \mathbb{N}}$ is a cauchy sequence.

Lemma 1.8. ([3-6]) If $(X, d)$ is a 2-metric space and 
sequence $\left\{x_{n}\right\}_{n \in \mathbb{N}} \rightarrow x \in X$, then

$$
\lim _{n \rightarrow+\infty} d\left(x_{n}, b, c\right)=d(x, b, c)
$$

for each $b, c \in X$.

Lemma 1.9. ([7,8]) Let $f, g: X \rightarrow X$ be weakly compatible. If $f$ and $g$ have a unique point of coincidence $w=f x=g x$, then $w$ is the unique common fixed point of $f$ and $g$.

\section{Main Results}

Denote by $\Gamma$ the set of functions $\gamma:[0,+\infty) \rightarrow[0,+\infty)$ satisfying the following:

( $\Gamma 1) \gamma$ is continuous; ( $Г 2) \gamma(t)<t$ for all $t>0$. Denote by $\Psi^{[1,2]}$ the set of functions

$$
\psi:[0,+\infty) \rightarrow[0,+\infty)
$$

satisfying the following:

( C 1) $\psi$ is continuous and non-decreasing; ( $C$ 2) $\psi(t)<t$ for all $t>0$.

Obviously, $\psi$ is stronger than $\gamma$.
Example 2.1. Define $\gamma(x):[0, \infty) \rightarrow[0, \infty)$ as follow:

$$
\gamma(x)= \begin{cases}\frac{1}{2} x, & \text { for } 0 \leq x \leq 1 \\ -\frac{1}{2} x+1, & \text { for } 1<x \leq \frac{4}{3} \\ \frac{1}{3}, & \text { for } x>\frac{4}{3}\end{cases}
$$

Obviously, $\gamma \in \Gamma$, but since $\gamma(1)=\frac{1}{2}>\frac{1}{3}=\gamma(2)$, so $\gamma \notin \Psi$.

The following is the main conclusion in this paper.

Theorem 2.2. Let $(X, d)$ be a 2-metric space,

$$
S, T, I, J: X \rightarrow X
$$

four mappings satisfying that

$$
S(X) \subset I(X) \text { and } T(X) \subset J(X) .
$$

Suppose that for each $x, y, a \in X$,

$$
d(S x, T y, a) \leq q \gamma\left(\max \left\{d(J x, I y, a), d(J x, S x, a), d(I y, T y, a), \frac{d(J x, T y, a)}{2}, \frac{d(I y, S x, a)}{2}\right\}\right)
$$

where $0<q<1$ and $\gamma \in \Gamma$. If one of

$$
S(X), T(X), I(X)
$$

and $J(X)$ is complete, then $T$ and $I, S$ and $J$ have an unique point of coincidence in $X$. Further, $\{I, T\}$ and $\{S, J\}$ are weakly compatible respectively, then $S, T, I, J$ have an unique common fixed point in
$X$.

Proof Take any element $x_{0} \in X$, then in view of the conditions $S(X) \subset T(X)$ and $T(X) \subset J(X)$, we can construct two sequences $\left\{x_{n}\right\}$ and $\left\{y_{n}\right\}$ as follows:

$y_{2 n}=S x_{2 n}=I x_{2 n+1}, \quad y_{2 n+1}=T x_{2 n+1}=J x_{2 n+2}, \quad n=0,1, \cdots$.

For any $n=0,1, \cdots$,

$$
\begin{aligned}
& d\left(y_{2 n}, y_{2 n+1}, a\right)=d\left(S x_{2 n}, T x_{2 n+1}, a\right) \\
& \leq q \gamma\left(\max \left\{d\left(J x_{2 n}, I x_{2 n+1}, a\right), d\left(J x_{2 n}, S x_{2 n}, a\right), d\left(I x_{2 n+1}, T x_{2 n+1}, a\right), \frac{d\left(J x_{2 n}, T x_{2 n+1}, a\right)}{2}, \frac{d\left(I x_{2 n+1}, S x_{2 n}, a\right)}{2}\right\}\right) \\
& =q \gamma\left(\max \left\{d\left(y_{2 n-1}, y_{2 n}, a\right), d\left(y_{2 n}, y_{2 n+1}, a\right), \frac{d\left(y_{2 n-1}, y_{2 n+1}, a\right)}{2}\right\}\right) .
\end{aligned}
$$

If

$$
\max \left\{d\left(y_{2 n-1}, y_{2 n}, a\right), d\left(y_{2 n}, y_{2 n+1}, a\right), \frac{d\left(y_{2 n-1}, y_{2 n+1}, a\right)}{2}\right\}=0
$$

for some $a \in X$, then $d\left(y_{2 n}, y_{2 n+1}, a\right)=0$, hence we have that

$$
d\left(y_{2 n}, y_{2 n+1}, a\right) \leq q d\left(y_{2 n-1}, y_{2 n}, a\right) .
$$

Hence we can assume now that

$$
\max \left\{d\left(y_{2 n-1}, y_{2 n}, a\right), d\left(y_{2 n}, y_{2 n+1}, a\right), \frac{d\left(y_{2 n-1}, y_{2 n+1}, a\right)}{2}\right\}>0
$$


for all $a \in X$.

If

$$
\max \left\{d\left(y_{2 n-1}, y_{2 n}, a\right), d\left(y_{2 n}, y_{2 n+1}, a\right), \frac{d\left(y_{2 n-1}, y_{2 n+1}, a\right)}{2}\right\}=d\left(y_{2 n}, y_{2 n+1}, a\right)
$$

for some $a \in X$, then (2) becomes that

$$
d\left(y_{2 n}, y_{2 n+1}, a\right) \leq q \gamma\left(d\left(y_{2 n}, y_{2 n+1}, a\right)\right)<q d\left(y_{2 n}, y_{2 n+1}, a\right),
$$

which is a contradiction since $q<1$. Hence we have that

$$
\max \left\{d\left(y_{2 n-1}, y_{2 n}, a\right), d\left(y_{2 n}, y_{2 n+1}, a\right), \frac{d\left(y_{2 n-1}, y_{2 n+1}, a\right)}{2}\right\}=\max \left\{d\left(y_{2 n-1}, y_{2 n}, a\right), \frac{d\left(y_{2 n-1}, y_{2 n+1}, a\right)}{2}\right\}
$$

for all $a \in X$.

If $d\left(y_{2 n-1}, y_{2 n}, a\right) \geq \frac{d\left(y_{2 n-1}, y_{2 n+1}, a\right)}{2}$ for some $a \in X$, then from (2),

$$
d\left(y_{2 n}, y_{2 n+1}, a\right) \leq q \gamma\left(d\left(y_{2 n-1}, y_{2 n}, a\right)\right)<q d\left(y_{2 n-1}, y_{2 n}, a\right) .
$$

If $d\left(y_{2 n-1}, y_{2 n}, a\right) \leq \frac{d\left(y_{2 n-1}, y_{2 n+1}, a\right)}{2}$ for some $a \in X$, then from (2),

$$
\begin{aligned}
d\left(y_{2 n}, y_{2 n+1}, a\right) & \leq q \gamma\left(\frac{d\left(y_{2 n-1}, y_{2 n+1}, a\right)}{2}\right)<\frac{q d\left(y_{2 n-1}, y_{2 n+1}, a\right)}{2} \\
& \leq \frac{q\left[d\left(y_{2 n-1}, y_{2 n}, y_{2 n+1}\right)+d\left(y_{2 n-1}, y_{2 n}, a\right)+d\left(y_{2 n}, y_{2 n+1}, a\right)\right]}{2} .
\end{aligned}
$$

If $d\left(y_{2 n-1}, y_{2 n}, y_{2 n+1}\right)>0$, then

$$
\begin{aligned}
& d\left(y_{2 n-1}, y_{2 n}, y_{2 n+1}\right)=d\left(S x_{2 n}, T x_{2 n+1}, y_{2 n-1}\right) \\
& \leq q \psi \gamma\left(\operatorname { m a x } \left\{d\left(J x_{2 n}, I x_{2 n+1}, y_{2 n-1}\right), d\left(J x_{2 n}, S x_{2 n}, y_{2 n-1}\right), d\left(I x_{2 n+1}, T x_{2 n+1}, y_{2 n-1}\right), \frac{d\left(J x_{2 n}, T x_{2 n+1}, y_{2 n-1}\right)}{2},\right.\right. \\
& \left.\left.\quad \frac{d\left(I x_{2 n+1}, S x_{2 n}, y_{2 n-1}\right)}{2}\right\}\right) \\
& =q \gamma\left(d\left(y_{2 n-1}, y_{2 n}, y_{2 n+1}\right)\right)<q d\left(y_{2 n-1}, y_{2 n}, y_{2 n+1}\right),
\end{aligned}
$$

which is a contradiction since $0<q<1$. hence $d\left(y_{2 n-1}, y_{2 n}, y_{2 n+1}\right)=0$.

So (4) becomes that

$$
\begin{aligned}
& d\left(y_{2 n}, y_{2 n+1}, a\right) \\
& <\frac{q\left[d\left(y_{2 n-1}, y_{2 n}, a\right)+d\left(y_{2 n}, y_{2 n+1}, a\right)\right]}{2} .
\end{aligned}
$$

Hence we obtain that

$$
d\left(y_{2 n}, y_{2 n+1}, a\right) \leq \frac{q}{2-q} d\left(y_{2 n-1}, y_{2 n}, a\right) .
$$

By (3) and (6), we obtain that

$$
\begin{aligned}
d\left(y_{2 n}, y_{2 n+1}, a\right) & \leq \max \left\{q, \frac{q}{2-q}\right\} d\left(y_{2 n-1}, y_{2 n}, a\right) \\
& =q d\left(y_{2 n-1}, y_{2 n}, a\right), \forall a \in X .
\end{aligned}
$$


Similarly, we can obtain that for each $n=0,1, \cdots$,

$$
d\left(y_{2 n+1}, y_{2 n+2}, a\right) \leq q d\left(y_{2 n}, y_{2 n+1}, a\right), \forall a \in X .
$$

Combining (7) and (8), we have that

$$
d\left(y_{n+1}, y_{n+2}, a\right) \leq q d\left(y_{n}, y_{n+1}, a\right), \forall a \in X .
$$

Hence $\left\{y_{n}\right\}$ is Cauchy sequence by Lemma 1.7.

Suppose that $I(X)$ is complete, then there exists $u \in I(X)$ and $v \in X$ such that

$$
\begin{gathered}
y_{2 n}=S x_{2 n}=I x_{2 n+1} \rightarrow u=I v . \\
d(u, T v, a) \leq d\left(y_{2 n}, T v, a\right)+d\left(u, y_{2 n}, a\right)+d\left(u, y_{2 n}, T v\right)=d\left(S x_{2 n}, T v, a\right)+d\left(u, y_{2 n}, a\right)+d\left(u, y_{2 n}, T v\right) \\
\leq q \gamma\left(\max \left\{d\left(J x_{2 n}, I v, a\right), d\left(J x_{2 n}, S x_{2 n}, a\right), d(I v, T v, a), \frac{d\left(J x_{2 n}, T v, a\right)}{2}, \frac{d\left(I v, S x_{2 n}, a\right)}{2}\right\}\right)+d\left(u, y_{2 n}, a\right)+d\left(u, y_{2 n}, T v\right) \\
=q \gamma\left(\max \left\{d\left(y_{2 n-1}, u, a\right), d\left(y_{2 n-1}, y_{2 n}, a\right), d(u, T v, a), \frac{d\left(y_{2 n-1}, T v, a\right)}{2}, \frac{d\left(u, y_{2 n}, a\right)}{2}\right\}\right)+d\left(u, y_{2 n}, a\right)+d\left(u, y_{2 n}, T v\right) .
\end{gathered}
$$

(If $S(X)$ is complete, then there exists $u \in S(X)$ $\subset I(X)$, hence the conclusions remains the same).

Since

$$
\begin{aligned}
d\left(y_{2 n+1}, u, a\right) \leq & d\left(y_{2 n+1}, y_{2 n}, a\right)+d\left(y_{2 n}, u, a\right) \\
& +d\left(y_{2 n}, y_{2 n+1}, u\right)
\end{aligned}
$$

and $\left\{y_{n}\right\}$ is Cauchy sequence and $\left\{y_{2 n}\right\} \rightarrow u$, we know that $\left\{y_{2 n+1}\right\} \rightarrow u$.
Let $n \rightarrow \infty$, then by Lemma 1.8, the above becomes

$$
d(u, T v, a) \leq q \gamma(d(u, T v, a)) .
$$

If $d(u, T v, a)>0$ for some $a \in X$, then we obtain that

$$
d(u, T v, a)<q d(u, T v, a)
$$

which is a contradiction since $0<q<1$. Hence $d(u, T v, a)=0$ for all $a \in X$, so $T v=u=I v$, i.e., $u$ is a point of coincidence of $T$ and $I$, and $v$ is a coincidence point of $T$ and $I$.

On the other hand, since $u=T v \in T(X) \subset J(X)$, there exists $w \in X$ such that $u=J w$. By (1), for any $a \in X$,

$$
\begin{aligned}
& d(S w, u, a) \leq d\left(S w, y_{2 n+1}, a\right)+d\left(y_{2 n+1}, u, a\right)+d\left(y_{2 n+1}, u, S w\right)=d\left(S w, T x_{2 n+1}, a\right)+d\left(y_{2 n+1}, u, a\right)+d\left(y_{2 n+1}, u, S w\right) \\
& \leq q \gamma\left(\max \left\{d\left(J w, I x_{2 n+1}, a\right), d(J w, S w, a), d\left(I x_{2 n+1}, T x_{2 n+1}, a\right), \frac{d\left(J w, T x_{2 n+1}, a\right)}{2}, \frac{\left(I x_{2 n+1}, S w, a\right)}{2}\right\}\right) \\
& +d\left(y_{2 n+1}, u, a\right)+d\left(y_{2 n+1}, u, S w\right) \\
& =q \gamma\left(\max \left\{d\left(u, y_{2 n}, a\right), d(u, S w, a), d\left(y_{2 n}, y_{2 n+1}, a\right), \frac{d\left(u, y_{2 n+1}, a\right)}{2}, \frac{d\left(y_{2 n}, S w, a\right)}{2}\right\}\right) \\
& \quad+d\left(y_{2 n+1}, u, a\right)+d\left(y_{2 n+1}, u, S w\right) .
\end{aligned}
$$

Let $n \rightarrow \infty$, then we obtain that

$$
d(S w, u, a) \leq q \gamma(d(S w, u, a)) .
$$

If $d(S w, u, a)>0$ for some $a \in X$, then the above becomes that

$$
\begin{aligned}
& d(S w, u, a)<q d(S w, u, a), \quad \begin{array}{l}
J, \text { then there exists } a \in X \text { such } \\
\text { and we have that }
\end{array} \\
& d(z, u, a)=d(S x, T v, a) \\
& \leq q \gamma\left(\max \left\{d(J x, I v, a), d(J x, S x, a), d(I v, T v, a), \frac{d(J x, T v, a)}{2}, \frac{d(I v, S x, a)}{2}\right\}\right) \\
& \quad q \gamma(d(z, u, a))<q d(z, u, a),
\end{aligned}
$$

which is a contradiction since $0<q<1$, so $d(S w, u, a)=$ for all $a \in X$. Hence $S w=u=J w$, i.e, $u$ is a point of coincidence of $S$ and $J$, and $w$ is a coincidence point of $S$ and $J$.

If $z=S x=J x$ is another point of coincidence of $S$ and $J$, then there exists $a \in X$ such that $d(z, u, a)>0$, 
which is a contradiction. So $d(z, u, a)=0$ for all $a \in X$, hence $z=u$, i.e, $u$ is the unique point of coincidence of $S$ and $J$. Similarly, we can prove that $u$ is also the unique point of coincidence of $T$ and $I$.

By Lemma 1.9, $u$ is the unique common fixed point $\{S, J\}$ and $\{T, I\}$ respectively, hence $u$ is the unique common fixed point of $S, T, I, J$.

If $J(X)$ or $T(X)$ is complete, then we can also use similar method to prove the same conclusion. We omit the part.

The following particular form of Theorem 2.2 for $\psi$-condition is the main result in [1]. The detailed proof can be found in [1].

Theorem 2.3. Let $(X, d)$ be a 2-metric space, $S, T, I, J: X \rightarrow X$ four mappings satisfying that $S(X)$ $\subset I(X)$ anwd $T(X) \subset J(X)$. Suppose that for each $x, y \in X$,

$$
d(S x, T y, a) \leq q \psi\left(\max \left\{d(J x, I y, a), d(J x, S x, a), d(I y, T y, a), \frac{d(J x, T y, a)}{2}, \frac{d(I y, S x, a)}{2}\right\}\right), \forall a \in X,
$$

where $0<q<1$ and $\psi \in \Psi$. If one of $S(X), T(X)$, $I(X)$ and $J(X)$ is complete, then $T$ and $I, S$ and $J$ have an unique point of coincidence in $X$. Further, $\{I, T\}$ and $\{S, J\}$ are weakly compatible respectively, then $S, T, I, J$ have an unique common fixed point in $X$.
Using Theorem 2.2, we can give many different type fixed point or common fixed point theorems. But we give only the next two contractive or quasi-contractive versions of Theorem 2.2 for two mappings.

Theorem 2.4. Let $(X, d)$ be a 2-metric space, $S, T: X \rightarrow X$ two mappings satisfying that for each $x, y, a \in X$,

$$
d(S x, T y, a) \leq q \gamma\left(\max \left\{d(x, y, a), d(x, S x, a), d(y, T y, a), \frac{d(x, T y, a)}{2}, \frac{d(y, S x, a)}{2}\right\}\right)
$$

where $0<q<1$ and $\gamma \in \Gamma$. If one of $S(X)$ and $T(X)$ is complete, then $S$ and $T$ have an unique common fixed point in $X$.
Theorem 2.5. Let $(X, d)$ be a complete 2-metric space, $I, J: X \rightarrow X$ two surjective mappings. If for each $x, y, a \in X$,

$$
d(x, y, a) \leq q \gamma\left(\max \left\{d(J x, I y, a), d(J x, x, a), d(I y, y, a), \frac{d(J x, y, a)}{2}, \frac{d(I y, x, a)}{2}\right\}\right),
$$

where $0<q<1$ and $\gamma \in \Gamma$. Then $I$ and $J$ have an unique common fixed point in $X$.

\section{REFERENCES}

[1] H. L. Jin and Y. J. Piao, "Four Mappings Satisfying $\Psi$-Contractive Type Condition and Having Unique Common Fixed Point on 2-Metric Spaces," Advances in Pure Mathematics, Vol. 3, No. 2, 2013, pp. 277-281. http://dx.doi.org/10.4236/apm.2013.32039

[2] B. Samet, M. Rajovic, R. Lazovi and R. Stoilikovic, "Common Fixed Point Results for Nonlinear Contractions in Ordered Partial Metric Spaces," Fixed Point and Applications, Vol. 71, No. 1, 2011. http://dx.doi.org/10.1186/1687-1812-2011-71

[3] S. L. Singh, "Some Contractive Type Principles on 2-Metric Spaces and Applications," Mathematics Seminar Notes (Kobe University), Vol. 7, No. 1, 1979, pp. 1-11.

[4] Y. J. Piao, "A Family of Quasi-Contractive Type NonCommutative Self-Maps Having an Unique Common Fixed Point in 2-Metric Spaces," Journal of Heilongjiang University (Science Edition), Vol. 23, No. 5, 2006, pp.
655-657.

[5] Y. J. Piao, "Unique Common Fixed Point for a Family of Self-Maps with Same Type Contractive Condition in 2-Metric Spaces," Analysis in Theory and Applications, Vol. 24, No. 4, 2008, pp. 316-320. http://dx.doi.org/10.1007/s10496-008-0316-9

[6] Y. J. Piao, "Unique Common Fixed Point for a Family of Self-Maps with Same Quasi-Contractive Type Condition in 2-Metric Space," Journal of Nanjing University (Mathematical Biquarterly), Vol. 27, No. 1, 2010, pp. 82-87.

[7] M. Abbas and G. Jungck, "Common Fixed Point Results for Noncommuting Mappings without Continuity in Cone Metric Spaces," Journal of Mathematical Analysis and Applications, Vol. 341, No. 1, 2008, pp. 416-420. http://dx.doi.org/10.1016/j.jmaa.2007.09.070

[8] Y. Han and S. Y. Xu, "New Common Fixed Point Results for Four Maps on Cone Metric Spaces," Applied Mathematics, Vol. 2, No. 9, 2011, pp. 1114-1118. http://dx.doi.org/10.4236/am.2011.29153

[9] C. D. Bari and P. Vetro, " $\phi$-Pairs and Common Fixed Points in Cone Metric Spaces," Rendiconti del Circolo Matematico Palermo, Vol. 57, 2008, pp. 279-285. 